\title{
Effets non linéaires et fluctuations quantiques
}

\author{
É. Giacobino \\ Laboratoire Kastler Brossel, Université Pierre et Marie Curie, École Normale \\ Supérieure et CNRS, 4 place Jussieu, 75232 Paris cedex 05, France
}

\begin{abstract}
Résumé : Ce cours présente une description des états comprimés du rayonnement et quelques méthodes de production de ces états comprimés, basées sur des principes d'optique non linéaires. Après une brève introduction à la quantification du champ de rayonnement, nous discutons les principales conséquences de la nature quantique du champ sur les fluctuations qui apparaissent dans les mesures. Nous étudions tout d'abord la modification des fluctuations quantiques dans un système optique linéaire comme une lame semi-transparente. Puis nous traitons trois systèmes qui permettent de manipuler les fluctuations quantiques du rayonnement : l'amplificateur paramétrique qui produit un champ "vide comprimé ", la cavité contenant un milieu possédant un indice non linéaire, qui produit un champ d'amplitude non nulle comprimé, et l'oscillateur paramétrique au-dessus du seuil qui produit des faisceaux jumeaux.
\end{abstract}

\section{INTRODUCTION}

Contrairement à ce qui se passe dans beaucoup d'autres domaines de la physique, il est relativement facile d'observer les fluctuations quantiques en optique. Lorsque la lumière est produite par un laser dont toutes les fluctuations d'origine mécanique, acoustique ou électrique ont été supprimées, les fluctuations quantiques donnent lieu à un bruit rémanent sur l'intensité lumineuse détectée. Ce bruit est connu sous le nom de bruit de grenaille (" shot noise" en anglais) ou bruit quantique standard. Dans les années récentes, l'amélioration des sources lasers et des détecteurs a fait que le bruit quantique standard est de plus en plus souvent atteint dans les mesures de précision, en spectroscopie ou en interférométrie, limitant ainsi la sensibilité de la détection.

On a longtemps pensé que le bruit quantique standard constituait une limite insurmontable. Mais au milieu des années 1980, des expériences ont montré que si les fluctuations quantiques étaient inévitables, leur effet sur les mesures pouvait être contourné. Avec des faisceaux lumineux dits "non classiques", on sait maintenant atteindre des réductions de bruit allant jusqu'à $90 \%$ au-dessous du bruit quantique standard. Nous nous intéresserons ici à deux types de systèmes produisant des faisceaux lumineux à bruit quantique réduit, les amplificateurs ou oscillateurs paramétriques utilisant des milieux non linéaires d'ordre $2\left(\chi^{(2)}\right)$ et des cavités optiques contenant des milieux non linéaires d'ordre 3 $\left(\chi^{(3)}\right)$. 


\section{FLUCTUATIONS QUANTIQUES ET ÉTATS COMPRIMES}

De manière simplifiée, on peut voir le bruit quantique observé sur l'intensité d'un faisceau laser idéal comme résultant de l'arrivée aléatoire des photons sur le détecteur. La distribution aléatoire des photons a une statistique de Poisson et peut se mesurer directement en régime de comptage de photons: Lorsque l'on mesure une intensité lumineuse dans un régime de fort flux où le comptage est impossible, on retrouve sur cette intensité la trace de la distribution des photons : à la valeur moyenne se superposent des fluctuations dont la valeur quadratique moyenne est proportionnelle à la racine carrée de l'intensité, ce qui est caractéristique de la distribution Poissonienne.

\subsection{Champ électromagnétique quantique}

Cependant l'image de l'arrivée aléatoire des photons ne rend pas compte de toutes les caractéristiques du bruit quantique, qui se manifeste non seulement sur l'intensité mais sur la phase ou sur les diverses composantes en quadrature du champ. Pour les représenter correctement, il faut faire appel à une théorie plus complète, où le champ électromagnétique est quantifié.

Une onde plane de fréquence $\omega_{\mathrm{l}}$ et de direction de propagation et de polarisation données, correspondant à un mode particulier du champ électromagnétique peut être décrite par son champ électrique [1]:

$$
E(t)=E_{1} \cos \omega_{L} t+E_{2} \sin \omega_{L} t
$$

où $E_{1}$ and $E_{2}$ sont les composantes en quadrature du champ. Quand le champ est quantifié, les deux composantes sont des opérateurs qui s'expriment en fonction des opérateurs de création et annihilation $a^{\dagger}$ et $a$ du mode, opérateurs qui ont pour effet d'ajouter ou de retirer un photon :

$$
E_{1}=\varepsilon_{0}\left(a+a^{\dagger}\right) \quad E_{1}=-i \varepsilon_{0}\left(a-a^{\dagger}\right)
$$

avec

$$
\varepsilon_{0}=\sqrt{h \omega_{L} / 2 \varepsilon_{0} V}
$$

où $\mathrm{V}$ est le volume dans lequel est quantifié le champ. $\varepsilon_{0}$ est l'ordre de grandeur du champ dans un état qui contient en moyenne 1 photon.

Le commutateur de $a^{\dagger}$ et $a$ étant : $\left[a, a^{\dagger}\right]=1, E_{1}$ et $E_{2}$ ne commutent pas. Ce sont des quantités conjuguées, de même que la position $q$ et l'impulsion $p$ d'une particule. En conséquence, elles ne peuvent pas être mesurées avec une précision infinie simultanément et la dispersion des résultats $\Delta E_{1}$ et $\Delta E_{2}$ obéit à une inégalité de Heisenberg :

$$
\Delta E_{1} \cdot \Delta E_{2} \geq \varepsilon_{0}^{2}
$$

On peut représenter le champ dans "l'espace des phases" où les coordonnées sont les deux composantes en quadrature, coefficients de $\sin \omega_{L} t$ et $\cos \omega_{L} t$ dans l'équation (1). Dans un tel diagramme, analogue à celui de la représentation de Fresnel, un champ classique est représenté par un vecteur dont le module et l'argument sont l'amplitude et la phase. Pour un champ quantique, le résultat des mesures sur $E_{1}$ et $E_{2}$ fluctue de quantités $\Delta E_{1}$ et $\Delta E_{2}$. L'extrémité du vecteur représentant le champ appartient à une surface dans l'espace des phases dont la dimension est supérieure à la limite donnée par l'équation (4). 
L'état le plus proche d'un champ classique est celui pour lequel les fluctuations sur les deux quadratures sont égales à la limite permise par l'équation (4). L'aire d'incertitude a alors une forme circulaire de diamètre $\varepsilon_{0}$ dans l'espace des phases, comme indiqué sur la figure 1 (sur cette figure, le champ et ses fluctuations ne sont pas à la même échelle ; un champ laser comprend de très nombreux photons, alors que les fluctuations ont pour ordre de grandeur le champ d'un photon). Un tel état d'incertitude minimum est appelé état cohérent. Les fluctuations qui lui correspondent sont les fluctuations quantiques standard.

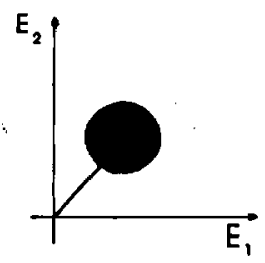

Figure 1 : Représentation d'un état cohérent dans l'espace des phases

Le champ correspondant à l'état de plus basse énergie d'un mode a une valeur moyenne nulle, mais, à cause du principe d'incertitude de Heisenberg, il ne peut avoir des fluctuations nulles. On peut montrer que cet état vide est un état cohérent particulier. Il est représenté dans l'espace des phases par un disque de diamètre $\varepsilon_{0}$ centré sur l'origine.

\subsection{Etats comprimés}

Pour réduire les fluctuations au-dessous de $\varepsilon_{0}$, il faut briser la symétrie entre les deux quadratures. On peut en effet diminuer $\Delta E_{1}$ pourvu que $\Delta E_{2}$ augmente au dessus de $\varepsilon_{0}$. Des états possédant cette propriété sont dits comprimés

Dans l'espace des phases, un état comprimé a une forme allongée, avec une dispersion plus petite sur une des composantes que sur l'autre. Les fluctuations peuvent être comprimées en amplitude c'est-à-dire parallèlement au champ moyen (figure 2, courbe a), ou en phase c'est-à-dire perpendiculairement au champ moyen (figure 2, courbe b), ou sur une quadrature quelconque, $E_{2}$ par exemple (figure 2 courbe c).

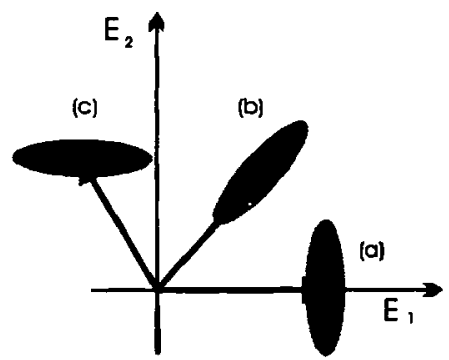

Figure 2 : Représentation d'un état comprimé dans l'espace des phases : (a) état comprimé en amplitude, (b) état comprimé en phase, (c) état comprimé en quadrature. 


\subsection{Mesures d'intensité}

Si le champ moyen est bien plus grand que les fluctuations, on peut écrire les fluctuations d'intensité dans le cadre d'une approximation linéaire

$$
\Delta I^{2}=4 E_{1}^{2} \Delta E_{1}^{2}
$$

où nous avons supposé que le champ moyen était aligné le long de l'axe $E_{1}$. Le bruit quantique standard correspond au cas où les fluctuations de d'amplitude sont égales à celles d'un champ cohérent : $\Delta E_{1}{ }^{2}=\varepsilon_{0}{ }^{2}$. Le bruit de photon est donc caractérisé par une variance proportionnelle à l'intensité moyenne :

$$
\left(\Delta^{2}\right)_{s t}=4 E_{1}^{2} \varepsilon_{0}
$$

Si l'on relie l'intensité au nombre de photons $\mathbf{N}$

on obtient

$$
I=\frac{2 \hbar \omega_{L}}{\varepsilon_{0} V} N
$$

$$
\left(\Delta N^{2}\right)_{s t}=<N>
$$

On retrouve la statistique de Poisson prévue par le modèle de l'arrivée aléatoire des photons. Le bruit d'intensité peut être réduit par compression des fluctuations de la composante $E_{1}$, c'est-à-dire ici de l'amplitude du champ. Plus généralement, dans la plupart des mesures optiques, seule une composante de quadrature du champ est concernée. On peut réduire le bruit sur la mesure en comprimant les fluctuations quantiques du champ sur la quadrature correspondante.

\subsection{Modèle de champ quantique pour un faisceau laser}

Jusqu'ici nous avons seulement considéré un champ strictement monomode et ses fluctuations à la même fréquence. De fait, les mesures sont sensibles aux fluctuations dans une certaine bande de fréquence, qui est l'inverse de la constante de temps utilisée. Nous sommes donc amenés à considérer des champs quasi monomodes, avec un mode de fréquence centrale $\omega_{\text {L }}$, dont la valeur moyenne du nombre de photons peut être importante, entouré de modes de fréquences voisines qui ne comportent pas ou presque pas de photons. Ces modes sont les modes de fluctuation qui ont des fréquences non nulles par rapport à la fréquence centrale.

Pour traiter ce cas, il est commode d'introduire un champ $\varepsilon(t)$ qui est une somme de Fourier des modes concernés, avec des fréquences de bruit $\omega$ faibles par rapport à la fréquence centrale $\omega_{\mathrm{L}}[2]$

$$
\begin{aligned}
& \mathcal{E}^{+}(t)=\varepsilon_{\omega_{L}} \int \frac{d \omega}{2 \pi} a_{\omega_{L}+\omega} \exp (-i \omega t) \exp \left(-i \omega_{L} t\right) \\
& \left.\mathcal{E}^{-}(t)=\varepsilon_{\omega_{L}} \int \frac{d \omega}{2 \pi} a_{\omega_{L}-\omega}^{\dagger} \exp (-i \omega t) \exp \left(+i \omega_{L} t\right)\right)
\end{aligned}
$$

La relation de commutation des opérateurs transformés de Fourier $a_{\omega_{L}+\omega^{\prime}}$ et $a^{\dagger} \omega_{L_{L}-\omega^{\prime}}$ est

$$
\left[a_{\omega_{L}+\omega^{\prime}} \cdot a_{\omega_{L}-\omega^{\prime}}^{\dagger}\right]=2 \pi \delta\left(\omega+\omega^{\prime}\right)
$$

où $\varepsilon_{\omega_{L}}$ est défini par 


$$
\varepsilon_{\omega_{\iota}}=\sqrt{\frac{\hbar \omega_{L}}{2 \varepsilon_{0} S c}}
$$

Dans cette expression, $S$ est la section du faisceau lumineux. On voit que la valeur moyenne du produit $\left\langle a_{\omega_{L}-\omega^{\prime}}^{\dagger} a_{\omega_{L}+\omega^{*}}\right\rangle$ est le nombre moyen de photons par unité de temps dans le faisceau. On peut montrer que la relation d'incertitude de Heisenberg pour la dispersion des composantes de quadrature, moyennée sur un certain temps de mesure $\mathrm{T}$ s'écrit maintenant

$$
\Delta E_{1} \Delta E_{2} \geq \frac{\varepsilon_{\omega_{2}}}{T}
$$

Cette expression est équivalente à l'expression (4) pour un mode de volume ScT. En utilisant cette relation pour calculer le bruit de photon (équation 6) on retrouve le résultat bien connu que le bruit est réduit si l'on moyenne les mesures sur des temps plus longs.

Dans la suite nous nous intéresserons plus spécifiquement aux composantes de Fourier des fluctuations du champ, et à leur spectre de bruit qui est directement accessible à l'expérience à l'aide d'un analyseur de spectre.

Pour montrer comment les fluctuations quantiques interviennent dans les mesures, nous allons tout d'abord étudier un système optique très simple, la lame séparatrice.

\section{BRUIT QUANTIQUE À LA SORTIE D'UNE LAME SÉPARATRICE}

Quand un faisceau laser passe à travers une lame semi-transparente (figure 3), en entrant par l'entrée $\mathrm{A}$, les deux sorties $\mathrm{C}$ et $\mathrm{D}$ voient leur bruit quantique augmenté. Cela peut se comprendre comme une conséquence de la nature corpusculaire de la lumière. Chaque photon a une probabilité $1 / 2$ d'être transmis ou réfléchi. Par suite du caractère aléatoire de ce processus de transmission/réflexion, il apparaît un bruit de partition qui est indépendant de la statistique de photon du faisceau entrant. On peut retrouver cette propriété de manière plus quantitative en calculant les fluctuations des champs aux deux sorties de la lame.

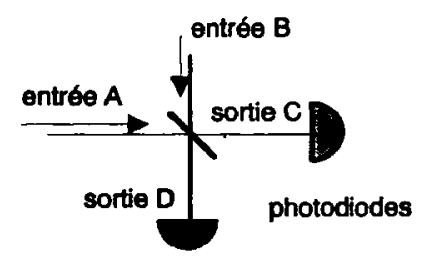

Figure 3 : Les champs entrant par les entrées A et B de la lame sont partiellement transmis et partiellement réfléchis et se recombinent sur les sorties $C$ et $D$

\subsection{Détection homodyne}

Considérons un dispositif souvent utilisé pour la " détection homodyne" qui comprend une lame séparatrice qui mélange les champs entrant par les voies A et $B$ et deux photodétecteurs sur les voies de sortie $C$ et $D$. Les coefficients de transmission et de réflexion en amplitude de la lame sont respectivement $t$ et $r$ (avec $r^{2}+t^{2}=1$ ). Les relations entre les champs quantiques entrants et sortants sont les mêmes que celles données par l'optique classique. 


$$
\begin{gathered}
E_{C}=t E_{A}+r E_{B} \\
E_{D}=-r E_{A}+t E_{B}
\end{gathered}
$$

Le signe - dans l'équation (13b) vient du déphasage de $\pi$ entre les réflexions air-verre et verreair. Considérons tout d'abord une lame semi-transparente. Dans ce cas, on a

$$
r=t=1 / \sqrt{2}
$$

Dans la suite, nous utiliserons la méthode " semi-classique" $[3,4,5]$ pour traiter les champs quantiques : les champs considérés sont la somme d'un champ classique de valeur égale à la valeur moyenne du champ quantique (éventuellement nulle) et d'un champ fluctuant dont la valeur quadratique moyenne est égale à celle des fluctuations quantiques. Ainsi, si l'on n'envoie aucun champ à l'entrée d'un système optique, il faudra supposer que le champ entrant est en fait le champ fluctuant du vide. Avec ces hypothèses on montre que l'on peut appliquer les lois de l'optique classique pour déterminer le champ sortant d'un système optique.

Nous utilisons maintenant ces principes pour calculer les intensités $I_{C}$ et $I_{D}$ des champs en sortie de la lame semi-transparente. En supposant que le champ $E_{A}$ est beaucoup plus intense que le champ $E_{B}$ nous obtenons

$$
\begin{aligned}
& I_{C}=1 / 2\left(E_{A}{ }^{2}+E_{A}\left(E_{B}+E_{B}{ }^{*}\right)\right) \\
& I_{D}=1 / 2\left(E_{A}{ }^{2}-E_{A}\left(E_{B}+E_{B}{ }^{*}\right)\right)
\end{aligned}
$$

où le champ $E_{A}$ a été supposé réel et où $\left(E_{B}+E_{B}{ }^{*}\right) / 2=E_{B I}$ est la composante de $E_{B}$ en phase avec l'oscillateur local $E_{A}$.

La différence $I$ des intensités $I_{C}$ et $I_{D} s$ 'écrit

$$
I=I_{C}-I_{D}=E_{A}\left(E_{B}+E_{B}^{*}\right)=2 E_{A} E_{B 1}
$$

Cette différence d'intensité est un signal résultant de l'homodynage par le champ $E_{A}$ (oscillateur local) de la composante de quadrature du champ $E_{B}$ alignée avec $E_{A}$. Lorsque l'on calcule les fluctuations de $I$, les fluctuations de l'oscillateur local disparaissent et on obtient

$$
\Delta I^{2}=4 E_{A}^{2} \Delta E_{B 1}^{2}
$$

Supposons maintenant que le champ entrant en $B$ est nul. Cela implique que les fluctuations du vide entrent en $B$ et sont homodynées par l'oscillateur local. Le bruit sur la différence d'intensité est alors donné par

$$
\Delta I^{2}=4 E_{A}^{2} \varepsilon_{0}^{2}
$$

Comparant ce résultat avec l'équation (6), on voit que ce bruit est le même que le bruit quantique standard du champ $E_{A}$ de l'oscillateur local. In est indépendant des fluctuations réelles de l'oscillateur local. Cette expérience procure donc une mesure directe du bruit quantique standard d'un champ quelconque.

Si maintenant on envoie sur l'entrée $B$ de la lame semi-transparente un champ qui n'est plus vide mais qui est constitué de "vide comprimé", le bruit sur $I$ n'est plus le bruit quantique standard. Si la quadrature comprimée de $E_{B}$ est alignée avec $E_{A}$ le bruit sur $I$ est inférieur au bruit quantique standard. Cette propriété a été utilisée dans les expériences de génération d'états comprimés qui sont décrites plus loin pour détecter la présence de ces états du champ. 


\subsection{Effets des pertes sur la lumière comprimée}

Les pertes optiques ont un effet destructeur sur la lumière comprimée. On peut modéliser les pertes optiques linéaires qui se produisent à la traversée d'un milieu matériel par une lame séparatrice ayant un coefficient de transmission en intensité $t^{2}=T$. Nous reprenons la lame de la figure 3 et nous supposons qu'un champ non nul entre par l'entrée $A$ tandis que le vide entre par l'entrée $B$. Les fluctuations d'amplitude du champ transmis $E_{C}$. (fluctuations en phase avec la valeur moyenne du champ, supposé réel) valent alors

$$
\Delta E_{C l}^{2}=t^{2} \Delta E_{A 1}^{2}+r^{2} \varepsilon_{0}^{2}
$$

La lame semi-transparente mélange les fluctuations des deux champs. Si les fluctuations du champ entrant sont égales au bruit quantique standard $\left(\Delta E_{A 1}^{2}=\varepsilon_{0}^{2}\right)$, on trouve que les fluctuations du champ sortant sont également égales au bruit quantique standard car $r^{2}+t^{2}=1$. Cependant, si le champ entrant a des fluctuations inférieures au bruit quantique standard, les pertes tendent à ramener ses fluctuations au bruit quantique standard et donc à détruire la compression de bruit. Notons que le même raisonnement est vrai pour un champ ayant un fort excès de bruit, qui est également ramené au bruit quantique standard.

Dans un système de détection, une efficacité quantique inférieure à 1 a le même effet, puisqu'un détecteur de rendement quantique $T$ peut être considéré comme un détecteur de rendement quantique 1 précédé d'une lame de coefficient de transmission $T$.

\section{GÉNÉRATION D'ÉTATS COMPRIMÉS DU RAYONNEMENT}

La plupart des méthodes utilisées pour générer des états comprimés reposent sur des processus d'optique non linéaire, qui peuvent être du second ordre $\left(\chi^{(2)}\right)$ ou du troisième ordre $\left(\chi^{(3)}\right)$. Le premier cas correspond au mélange à 3 ondes : un milieu à coefficient $\chi^{(2)}$ non nul, irradié par un faisceau laser de pompe à la fréquence $\omega_{0}$ émet deux ondes dites signal et complémentaire (idler), de fréquence $\omega_{1}$ et $\omega_{2}$ vérifiant la condition de conservation de l'énergie, $\omega_{1}+\omega_{2}=\omega_{0}$. Un photon pompe disparaît alors que deux photons, signal et complémentaire, sont créés.

Le second cas correspond au mélange à 4 ondes : dans un milieu à coefficient $\chi^{(3)}$, deux photons de l'onde pompe à la fréquence $\omega_{0}$ disparaissent tandis que deux photons sont créés dans les ondes signal et complémentaire aux fréquences $\omega_{1}$ et $\omega_{2}$. La condition de conservation de l'énergie requiert : $\omega_{1}+\omega_{2}=2 \omega_{0}$. Ces deux types de systèmes peuvent être utilisés soit dans le régime non dégénéré où les fréquences $\omega_{1}$ et $\omega_{2}$ et/ou les polarisations sont différentes, soit dans le régime dégénéré où les fréquences sont égales et les polarisations identiques. Dans ce dernier cas, les champs signal et complémentaire se confondent en un seul et même champ.

La première observation de compression des fluctuations lumineuses a été effectuée en 1985, dans une expérience reposant sur le mélange à 4 ondes dégénéré dans de la vapeur de sodium [6]. La non-linéarité était exaltée en plaçant le milieu atomique dans une cavité. En 1986, de la lumière comprimée a été produite dans un système utilisant un milieu non linéaire du second ordre dans une cavité [7]. Nous décrirons dans la suite la génération de lumière comprimée dans un milieu à $\chi^{(2)}$ utilisé en régime dégénéré, puis dans un milieu $\chi^{(3)}$ utilisé comme un milieu possédant un indice non linéaire (effet Kerr) $[8,9]$ et enfin le cas du mélange à trois ondes non dégénéré, qui permet de produire un autre type de lumière non classique, les “faisceaux jumeaux " [10]. 


\subsection{Production de lumière comprimée par génération paramétrique dégénérée}

\subsubsection{Théorie}

Nous allons traiter la compression du bruit quantique dans un milieu non linéaire par la méthode semi-classique évoquée plus haut : nous calculons les champs par les équations de l'électromagnétisme classique, en tenant compte du fait que les champs entrants ont des fluctuations aléatoires qui " simulent" celles des champs quantiques. En particulier quand le champ entrant est nul classiquement, nous considérerons que ce sont les fluctuations du vide qui entrent dans le système. Pour les interactions paramétriques de type $\chi^{(2)}$ et $\chi^{(3)}$, on peut montrer [11] que ce traitement est complètement équivalent au traitement quantique standard.

Nous considérons un milieu non linéaire de coefficient $\chi^{(2)}$ non nul, éclairé par un laser de pompe à la fréquence $\omega_{0}$ placé dans une cavité optique résonante pour le sub-harmonique $\omega_{1}=\omega_{0} / 2$. Un tel système peut osciller comme un laser si le laser pompe a une intensité supérieure à un certain seuil [12]. Ici, nous supposerons que la puissance de pompe est assez faible pour que la cavité reste au-dessous du seuil d'oscillation. L'équation qui donne la modification de l'amplitude (complexe) du champ signal $\alpha_{1}$ sur un tour de cavité s'écrit :

$$
\tau \frac{\partial \alpha_{1}}{\partial t}=-\gamma \alpha_{1}+\eta \alpha_{1}^{*}+t \alpha_{1}^{i n}
$$

où $\tau$ est le temps d'aller-retour dans la cavité, $\gamma$ est l'amortissement du champ dans la cavité (sans dimension) qui est relié aux coefficients de transmission (supposé faible) et de réflexion du miroir de sortie $r$ et $t$ par

$$
\gamma=1-r=t^{2} / 2
$$

$\eta$ est le gain paramétrique, qui est proportionnel à l'intensité du champ pompe. On a supposé que le champ pompe n'est pas modifié à la traversée du milieu (pas de "déplétion" de la pompe) et que l'accord de phase est réalisé entre onde pompe et sub-harmonique. Soulignons que le gain paramétrique sur $\alpha_{1}$ fait intervenir $\alpha_{1}{ }^{*}$, ce qui est caractéristique de l'amplification paramétrique.

Le champ $\alpha_{1}^{\text {in }}$ est le champ entrant dans le système dans le mode qui respecte la condition d'accord de phase. Ici ce champ est le champ du vide [3].

Nous exprimerons la solution en termes des composantes de quadrature

$$
q_{1}=\left(\alpha_{1}+\alpha_{1}^{*}\right) / \sqrt{2} \quad p_{1}=\left(\alpha_{1}-\alpha_{1}^{*}\right) / \sqrt{2} i
$$

avec des notations similaires pour $q_{l}^{\text {in }}$ et $q_{l}^{\text {in }}$.

Nous utilisons le formalisme des champs quasi monomodes introduits à la section 2.4, et nous remplaçons $\alpha_{1}$ par son expression comme somme de Fourier de modes de fréquence $\omega_{1}+\omega$ où $\omega$ est une fréquence de bruit. La solution de l'équation (20) et de 'son complexeconjugué s'écrit

$$
q_{1}(\omega)=\frac{t q_{1}^{i n}(\omega)}{\gamma-\eta+i \omega \tau} \quad p_{1}(\omega)=\frac{t p_{1}^{i n}(\omega)}{\gamma+\eta+i \omega \tau}
$$

Par ailleurs, le champ sortant $\alpha_{1}{ }^{\text {out }}$ est relié au champ dans la cavité $\alpha_{1}$ et au champ entrant $\alpha_{1}^{\text {in }}$ par une relation de réflexion-transmission semblable à l'équation (13b)

$$
\alpha_{1}^{\text {out }}=t \alpha_{1}-\alpha_{1}^{\text {in }}
$$


où le coefficient de réflexion a été remplacé par 1. Ainsi le champ sortant est égal à la somme du champ dans la cavité transmis par le miroir et des fluctuations du vide réfléchies par le miroir. Utilisant les équations (23) et (24), on obtient

$$
q_{1}^{o \omega}(\omega)=\frac{\gamma+\eta-i \omega \tau}{\gamma-\eta+i \omega \tau} q_{1}^{\text {in }}(\omega) \quad p_{1}^{o \omega}(\omega)=\frac{\gamma-\eta-i \omega \tau}{\gamma+\eta+i \omega \tau} p_{1}^{\text {in }}(\omega)
$$

Ces équations montrent clairement que le champ du vide entrant a été modifié : la quadrature $q$, est amplifiée tandis que la quadrature $p_{I}$ est “dé-amplifiée " c'est-à-dire comprimée et tend vers 0 pour la fréquence de bruit nulle et pour $\gamma=\eta$, ce qui correspond au seuil d'oscillation de la cavité. La plage de fréquence de bruit sur laquelle apparaît la compression est de l'ordre de la largeur de la cavité. Au-delà de cette valeur, le bruit revient au bruit quantique standard suivant une fonction lorentzienne. Le champ du vide est ainsi comprimé par ce système. On appelle souvent l'état de sortie “vide comprimé ", bien que ce champ ne soit plus vide à proprement parler [13].

\subsubsection{Expériences}

Le montage expérimental [7] employé pour produire le " vide comprimé " comprend un cristal non linéaire de niobate de lithium dopé oxyde de magnésium $\left(\mathrm{MgO}: \mathrm{NiNbO}_{3}\right)$ pompé par un laser à YAG monomode doublé à $0.532 \mu \mathrm{m}$. Le cristal est placé dans une cavité résonante pour le champ sub-harmonique à $1.06 \mu \mathrm{m}$ (figure 4). L'accord de phase est réalisé pour ces longueurs d'ondes en chauffant le cristal. Le champ sortant de la cavité est analysé à l'aide de la détection homodyne décrite à la section 3.2, où l'oscillateur local est constitué par une partie du fondamental du laser de pompe à $1.06 \mu \mathrm{m}$. Le signal de différence des photodiodes de la détection homodyne est envoyé dans un analyseur de spectre.

Le signal observé à une fréquence de bruit fixée dépend de la phase relative de l'oscillateur local et du champ émis par la cavité. L'expérience montre qu'il oscille autour du niveau du bruit quantique standard en fonction de la phase de l'oscillateur local. La compression de bruit ainsi obtenue augmente lorsque la puissance de pompe se rapproche du seuil d'oscillation de la cavité. La réduction de bruit optimum obtenu avec ce type montage est de l'ordre de $80 \%$ au-dessous du bruit quantique standard $[14,15]$.

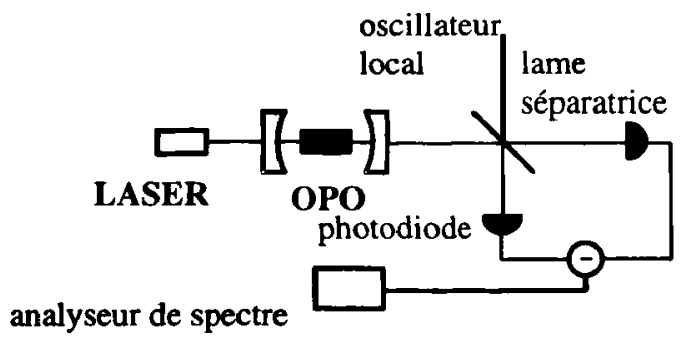

Figure 4 : Schéma expérimental utilisant la génération paramétrique dans une cavité pour prođuire du vide comprimé. Le vide comprimé est analysé en le recombinant avec un oscillateur local dans un détecteur homodyne fait d'une lame séparatrice 50/50 et de deux photodétecteurs 


\subsection{Production de lumière comprimée par un milieu possédant un indice non linéaire}

Dans cette section, nous allons considérer un système utilisant un milieu non linéaire du troisième ordre. L'interaction d'un champ avec un tel milieu produit une compression des fluctuations après propagation sur une distance suffisante. Ce phénomène a été mis en évidence dans des fibres optiques en silice [16] et dans des semi-conducteurs [17]. Les expériences avec des impulsions très courtes ou des solitons sont particulièrement intéressantes [18]. Toutefois, l'effet est en général plus facile à observer quand le milieu non linéaire est placé dans une cavité. Quand ce système est éclairé par un laser assez intense, il peut donner lieu au phénomène de bistabilité optique. Les premières propositions pour produire de la lumière comprimée reposaient sur une telle cavité non linéaire au voisinage du seuil de bistabilité $[8,9]$.

\subsubsection{Théorie}

Le traitement des fluctuations quantiques du champ dans une cavité non linéaire s'appuie sur la théorie semi-classique développée à la section 4.1.1 [4]. Nous considérons une cavité comprenant un miroir complètement réfléchissant et un miroir de couplage (cavité à une entrée-sortie). Classiquement, les champs entrant et sortant de la cavité sont reliés par

$$
\begin{aligned}
& t \alpha^{\text {in }}=\left(1-r e^{-i \Phi}\right) \alpha \\
& t \alpha^{\text {out }}=\left(e^{-i \Phi}-r\right) \alpha
\end{aligned}
$$

où $r$ et $t$ sont les coefficients de réflexion et de transmission en amplitude du miroir de couplage et $\Phi$ le déphasage total sur un aller et retour dans la cavité, qui comprend le déphasage linéaire et le déphasage non linéaire dû au milieu non linéaire (milieu Kerr) :

$$
\Phi=\Phi_{0}+K|\alpha|^{2}
$$

La transformation $\alpha_{1}{ }^{\text {in }} \rightarrow \alpha_{1}^{\text {out }}$ est une rotation dans l'espace des phases d'un angle qui dépend de l'intensité du champ dans la cavité. De ce fait tous les points de la distribution de probabilité montrée sur la figure 5 ne tournent pas du même angle et la forme de cette distribution est modifiée. Comme la surface totale est conservée dans la rotation, une élongation dans une direction entraîne une diminution dans une autre direction, et donne la compression recherchée.

Pour calculer le spectre de compression, nous écrivons comme précédemment l'équation d'évolution du champ dans la cavité. Nous supposons que la cavité a une bonne finesse et que le déphasage $\Phi$ est faible de sorte que l'on peut développer l'exponentielle de l'équation (26). L'amortissement du champ dans la cavité (sans dimension) $\gamma$ est relié aux coefficients $r$ et $t$ par l'équation (21). En conséquence, le coefficient de transmission $t$ peut s'exprimer comme

$$
t=\sqrt{2 \gamma}
$$




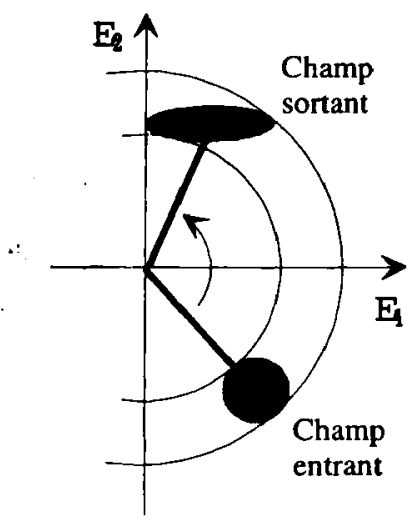

Figure 5 : Représentation dans l'espace des phases de la modification d'un champ cohérent après passage dans une cavité contenant un milieu non linéaire. Le champ subit une rotation dépendant de l'intensité qui produit une distorsion de la distribution de probabilité.

Le temps d'aller et retour dans la cavité est $\tau$ et le taux de déclin du champ dans la cavité est $\gamma / \tau$. Le milieu non linéaire est dans un premier temps supposé idéal (sans pertes). Dans ces conditions l'équation d'évolution du champ s'écrit

$$
\tau \frac{\partial \alpha(t)}{\partial t}=-\left(\gamma+i \Phi_{0}+i K|\alpha(t)|^{2}\right)+\sqrt{2 \gamma} \alpha^{i n}(t)
$$

où le champ entrant $\alpha_{1}^{\text {in }}$ (d'amplitude moyenne non nulle dans ce cas) est relié au champ sortant $\alpha_{1}{ }^{\text {out }}$ par

$$
\alpha^{\text {out }}(t)=\sqrt{2 \gamma} \alpha(t)-\alpha^{\text {in }}(t)
$$

Contrairement au cas de la section 4.1 où nous traitions des équations linéaires, nous avons maintenant une équation différentielle non linéaire. Considérant que les fluctuations sont faibles par rapport au champ moyen, nous résolvons tout d'abord l'équation stationnaire pour les champs moyens, puis nous différentions l'équation (29) autour des valeurs moyennes pour trouver les fluctuations quantiques. En prenant ensuite la transformation de Fourier comme précédemment, nous obtenons

$$
\begin{gathered}
\left(\gamma+i \Phi_{0}+2 i K\left|\alpha_{0}\right|^{2}-i \omega t\right) \delta \alpha(\omega)+i K \alpha_{0}^{2} \delta \alpha^{*}(\omega)=\sqrt{2 \gamma} \delta \alpha^{i n}(\omega) \\
\left(\gamma-i \Phi_{0}-2 i K\left|\alpha_{0}\right|^{2}-i \omega t\right) \delta \alpha^{*}(\omega)-i K \alpha_{0}^{* 2} \delta \alpha(\omega)=\sqrt{2 \gamma} \delta \alpha^{i n^{*}}(\omega)
\end{gathered}
$$

où les $\delta \alpha$ sont les fluctuations quantiques des champs, $\alpha_{0}$ est la valeur moyenne stationnaire et $\delta \alpha^{\text {in }}$ correspond aux fluctuations quantiques du champ entrant, supposé ici égal au bruit quantique standard. Les équations (31) présentent comme dans le cas de la génération paramétrique une dépendance en phase qui produit la compression de bruit. On trouve que la compression est totale pour des points de fonctionnement au voisinage des points tournants de la courbe de bistabilité. Nous ne donnerons pas ici l'expression finale qui a une forme plus compliquée que les équations (25). On trouve aussi que la plage de fréquence de bruit sur laquelle apparaît la compression est de l'ordre de la largeur de la cavité. Si on introduit des pertes dans la cavité, la compression de bruit est dégradée. 


\subsubsection{Expériences}

La génération d'états comprimés a été observée dans un système comprenant des atomes à deux niveaux dans une cavité. Pour éliminer l'effet Doppler, on utilise soit un jet atomique perpendiculaire à la cavité [19], soit des atomes refroidis par laser [20].

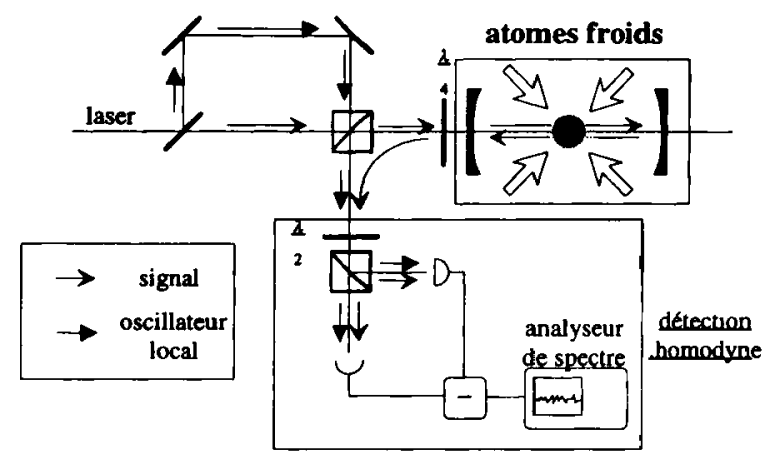

Figure 6 : Schéma expérimental montrant la production de lumière comprimée à l'aide d'une cavité contenant des atomes refroidis par laser qui constituent le milieu non linéaire.

Dans ce cas, le milieu ne peut plus être considéré comme un milieu Kerr parfait, car les atomes sont excités au voisinage d'une résonance atomique et des effets supplémentaires comme l'émission de lumière de fluorescence doivent être pris en compte dans l'étude théorique [21].

L'expérience comportant des atomes refroidis par laser est schématisée sur la figure 6. Les atomes sont illuminés par un ensemble de 6 faisceaux lasers se contre-propageant dans les 3 directions de l'espace et placés dans un gradient de champ magnétique. L'ensemble forme un piège magnéto-optique qui piège et refroidit les atomes à une température de l'ordre du $\mathrm{mK}$. Les atomes sont entourés par une cavité optique proche de résonance pour le faisceau laser à comprimer. La fréquence de ce faisceau laser est décalée de la résonance atomique d'environ 10 largeurs de raie. La puissance de ce faisceau est très faible, environ $10 \mu \mathrm{W}$, sur un waist de cavité de $1 \mathrm{~mm}^{2}$. La sortie de la cavité comporte une diode optique formée par un polariseur et une lame $\lambda / 4$, qui permet de séparer le faisceau réfléchi par la cavité du faisceau entrant. À la sortie de la cavité, le faisceau est mélangé avec un oscillateur local de puissance $10 \mathrm{~mW}$ issu du même laser, et détecté par un système analogue à celui présenté à la section 4.1.1. La compression de bruit obtenue avec ce montage est de 40\% [20].

\subsection{Production de faisceaux jumeaux par génération paramétrique non dégénérée}

Comme nous l'avons indiqué plus haut, dans la génération paramétrique, deux photons, signal et complémentaire, sont émis en même temps, tandis qu'un photon pompe est annihilé. Les photons sont donc créés par paires, ce qui produit une corrélation au niveau quantique entre les intensités des faisceaux correspondants. Ces faisceaux, qui diffèrent par la fréquence et/ou la polarisation peuvent être séparés au moyen d'un prisme ou d'un polariseur, et leurs intensités comparées à l'aide de deux photodétecteurs. La corrélation quantique a été observée soit en simple passage [22] dans un cristal soit en cavité. 
Une cavité résonante pour les ondes signal et complémentaire placée autour du cristal a l'avantage de renforcer l'effet non linéaire. Dans ce cas, la cavité est pompée par un laser intense, au-dessus du seuil d'oscillation. Elle émet deux faisceaux jumeaux, qui présentent un fort degré de corrélation quantique. Cependant la cavité introduit une certaine décorrélation entre les deux faisceaux. En effet, les deux photons d'une paire ne sortent pas nécessairement de la cavité en même temps. Si le temps de mesure est faible devant le temps de stockage dans la cavité, les photons d'une même paire ne seront en général pas détectés tous les deux. En revanche, si le temps de mesure est long devant le temps de stockage de la cavité, toutes les paires sont détectées, l'on s'attend à ce que les deux intensités ainsi mesurées présentent un fort degré de corrélation. Quantitativement, la différence des intensités doit avoir un bruit inférieur au bruit quantique standard (qui serait celui de la différence de deux faisceaux non corrélés, ayant tous deux un bruit égal au bruit quantique standard). Cela correspond, dans le domaine des fréquences, c'est-à-dire pour le signal donné par un analyseur de spectre, à un spectre de bruit au-dessous du bruit quantique standard à basse fréquence et qui remonte au bruit quantique standard pour des fréquences supérieures à la bande passante de la cavité.

\subsubsection{Théorie}

Pour déterminer les fluctuations quantiques à la sortie d'un oscillateur paramétrique non dégénéré au-dessus du seuil, nous employons à nouveau la méthode semi-classique de la section 4.1.1 [23]. Cependant dans ce cas, il faut tenir compte de la déplétion de la pompe. Les équations sont alors non linéaires et nous utilisons la méthode de linéarisation décrite à la section 4.2.1. Comme dans l'équation (20) les termes sources pour les champ signal $\alpha_{1}$ et complémentaire $\alpha_{2}$ sont les fluctuations du vide entrant par le miroir de couplage. Le terme source pour le champ pompe $\alpha_{0}$ est le champ cohérent $\alpha_{0}^{\text {in }}$ qui a une amplitude moyenne non nulle et des fluctuations égales à celles du vide. Les équations d'évolution des trois champs dans la cavité s'écrivent

$$
\begin{gathered}
\tau \frac{\partial \alpha_{1}}{\partial t}=-\gamma \alpha_{1}-\chi \alpha_{2}^{*} \alpha_{0}+\sqrt{2 \gamma} \alpha_{1}^{i n} \\
\tau \frac{\partial \alpha_{2}}{\partial t}=-\gamma \alpha_{2}-\chi \alpha_{1}^{*} \alpha_{0}+\sqrt{2 \gamma} \alpha_{2}^{i n} \\
\tau \frac{\partial \alpha_{0}}{\partial t}=-\gamma_{0} \alpha_{0}-\chi \alpha_{1} \alpha_{2}+\sqrt{2 \gamma_{0}} \alpha_{0}^{i n}
\end{gathered}
$$

où $\chi$ est le coefficient non linéaire du second ordre du cristal, $\gamma$ et $\gamma_{0}$ sont les coefficients d'amortissement des champs signal et complémentaire d'une part, pompe d'autre part et les autre notations sont analogues à celles de l'équation (20). Au-dessus d'une puissance de pompe seuil donnée par

$$
\mid\left.\alpha_{0}^{\text {in }}(\text { seuil })\right|^{2}=\gamma_{0} \gamma^{2} / 2 \chi^{2}
$$

les valeurs moyennes stationnaires des champs au-dessus du seuil sont

$$
\begin{gathered}
\alpha_{0}=-\gamma / \chi \\
\left|\alpha_{1}\right|^{2}=\left|\alpha_{2}\right|^{2}=\left(\gamma_{0} \gamma / \chi^{2}\right)(\sigma-1)
\end{gathered}
$$

où le paramètre $\sigma$ vaut

$$
\sigma=\sqrt{2 \chi^{2} / \gamma_{0} \gamma^{2}}\left|\alpha_{0}^{i n}\right|
$$


Comme précédemment, on linéarise les équations autour des valeurs stationnaires, on effectue une transformation de Fourier et on introduit les composantes de quadrature des champs, définies par

$$
q_{i}=\left(\alpha_{i}+\alpha_{i}^{*}\right) / \sqrt{2} \quad p_{i}=\left(\alpha_{i}-\alpha_{i}^{*}\right) / \sqrt{2} i
$$

où $i=1,2,0$, et les différences

$$
q_{-}=\left(q_{1}-q_{2}\right) / \sqrt{2} \quad p_{-}=\left(p_{1}-p_{2}\right) / \sqrt{2}
$$

Nous choisissons les phases de telle sorte que les champs moyens $\alpha_{1}$ et $\alpha_{2}$ soient réels. Les fluctuations de type $\delta q$ sont alors de fluctuations d'amplitude alors que les $\delta p$ sont des fluctuations de phase. Les fluctuations sur la différence des amplitudes sont données par

$$
\tau \frac{\partial \delta q_{-}}{\partial t}=-\gamma \delta q_{-}+\left(\chi q_{0} / \sqrt{2}\right) \delta q_{-}+\sqrt{2 \gamma} \delta q_{-}^{i n}
$$

Nous voyons qu'elles ne sont pas couplées aux autres quantités et qu'elles vérifient une équation analogue à l'équation 20. En utilisant la relation d'entrée-sortie (30), on obtient

$$
\delta q_{-}^{\text {out }}(\omega)=\frac{\gamma+\chi q_{0} / \sqrt{2}-i \omega \tau}{\gamma-\chi q_{0} / \sqrt{2}+i \omega \tau} \delta q_{-}^{\text {in }}(\omega)
$$

Puisque $\gamma=-\chi q_{0} / \sqrt{2}$ on voit que les fluctuations sur la différence des amplitudes et sur la différence des intensités qui lui est proportionnelle, tendent vers 0 pour des fréquences nulles. Le spectre de bruit, donné par le module carré de l'équation (39), avec le bruit quantique standard (bruit entrant) normalisé à 1 , est de forme lorentzienne.

$$
S_{t_{1}-t_{2}}(\omega)=\frac{\omega^{2} \tau^{2}}{\omega^{2} \tau^{2}+4 \gamma^{2}}
$$

La compression de bruit obtenue ici est extrêmement robuste, puisqu'elle ne dépend ni de l'intensité des faisceaux signal et complémentaire, ni du bruit de pompe. Le seul processus qui dégrade la compression est constitué par les pertes optiques. Si $\gamma^{\prime}$ est le coefficient de perte à l'intérieur de la cavité, on montre que la compression est dégradée par le facteur $\gamma /\left(\gamma+\gamma^{\prime}\right)$. La compression prévue à fréquence nulle n'est plus parfaite, mais elle peut rester très importante avec un cristal de bonne qualité optique.

\subsubsection{Expériences}

Le dispositif expérimental utilisé dans les références [10,24] comprend un cristal non linéaire de KTP, pompé par un laser à argon monomode ou par un laser à YAG doublé et placé dans une cavité optique résonante pour les champs signal et complémentaire, et aussi pour la pompe, mais avec une finesse moindre (figure 7).

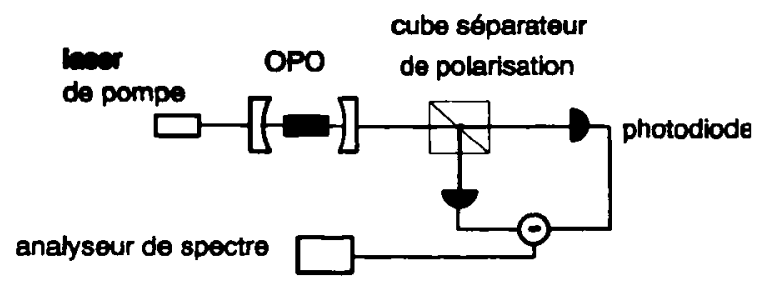

Figure 7 : Schéma du montage expérimental utilisé pour la production de faisceaux jumeaux. 
Le cristal étant de type II, les champs signal et complémentaire ont des polarisations orthogonales et sont facilement séparés à la sortie de la cavité par un prisme polariseur. Les intensités des deux champs sont détectées par des photodiodes et soustraites. La différence est envoyée sur un analyseur de spectre. Le bruit sur la différence d'intensité est réduit de $86 \%$ par rapport au bruit quantique standard. La réduction de bruit est effective sur une bande de fréquence qui est de l'ordre de la largeur de la cavité.

\section{CONCLUSION}

La compression du bruit quantique a fait de gros progrès dans les dernières années. On sait maintenant réduire le bruit quantique par un ordre de grandeur. D'une manière générale, l'expérience montre que les systèmes non linéaires du second ordre donnent des taux de compression supérieurs aux systèmes du troisième ordre. Cela provient du fait qu'il existe d'excellents cristaux non linéaires possédant un fort $\chi^{(2)}$ sans effets parasites (niveau résonant, pertes par absorption), tandis que les milieux $\chi^{(3)}$ ont des non-linéarités plus faibles, sauf si l'on se place au voisinage d'une résonance, ce qui introduit des effets parasites de pertes et d'excès de bruit.

Un processus complètement différent a été utilisé pour produire des champs dont l'intensité est comprimée. Il ne s'agit pas d'un effet optique non linéaire mais d'un effet laser. Le bruit à la sortie du laser est réduit par la régulation du bruit de pompe. En pratique, cette idée a été réalisée sur des lasers à semi-conducteur ayant un courant électrique de pompage stabilisé. Si l'efficacité quantique de la conversion électron-photon est assez grande, on peut montrer que le laser émet un flux de photons qui est lui-même stabilisé, ce qui donne un bruit d'intensité inférieur au bruit quantique standard [25]. Cette technique a permis d'atteindre des taux de compression de l'ordre de $75 \%$ [26].

Les systèmes utilisés dans certaines des expériences utilisent des systèmes atomiques assez encombrants ou des cristaux massifs. Un premier pas sur la voie de la miniaturisation a été effectué avec l'utilisation de cavités monolithiques (les miroirs sont déposés directement sur le cristal) comprenant des cristaux de très petite taille [15]. Récemment des taux de compression importants ont été obtenus dans des fibres optiques sur des solitons [27]. Il est également envisageable d'employer des guides intégrés non linéaires à faibles pertes.

Les applications envisagées pour la compression de bruit quantique concernent la physique fondamentale avec les mesures de précision (spectroscopie de haute sensibilité [28] ou interférométrie [29] destinée à la détection des ondes gravitationnelles). La compression du bruit lumineux devrait aussi trouver des utilisations plus appliquées dans des domaines comme les télécommunications [30].

\section{Références}

[1] Cohen-Tannoudji C., Dupont-Roc J., Grynberg G. (1988), Processus d'interaction entre photons et atomes, Interéditions/ Editions du CNRS, Paris (Atom-photon interactions (1991) Wiley, New-York).

[2] Fabre C. in Quantum Fluctuations (Eds. S. Reynaud, E. Giacobino et J. Zinn-Justin, North Holland Elsevier, Amsterdam, 1997)

[3] Reynaud S. et Heidmann A., Opt. Commun. 71, 209 (1989)

[4] Reynaud S., Fabre C., Giacobino E., Heidmann A., Phys. Rev. A40, 1440 (1989). 
[5] Reynaud S., Heidmann A., Giacobino E., Fabre C., in Progress in Optics XXX (Ed. E. Wolf, Elsevier New-York 1992)

[6] Slusher R.E, Hollberg L.W., Yurke B., Mertz J.C. et Valley J.F., Phys. Rev.Lett. 55, 2409 (1985); Slusher R.E., Yurke B., Grangier P., LaPorta A., Walls D.F. et Reid M., J Optical Society Am. 4, 1453 (1987).

[7] Wu L.A., Kimble H.J., Hall J.L. et Wu H., Phys. Rev. Lett. 57, 2520 (1986); Wu L.A., Min Xiao et Kimble H.J., J. Opt. Soc. Am. B4, 1465 (1988).

[8] Lugiato L., Strini G., Optics Commun. 41, 67 and 374 (1982).

[9] Collett M.J., Walls D.F., Phys. Rev. A 32, 2887 (1985).

[10] Heidmann A., Horowicz R.J., Reynaud S., Giacobino E., Fabre C. et Camy G., Phys. Rev. Lett. 59, 2555 (1987).

[11] Fabre C., Giacobino E., HeidmannA., Lugiato L., Reynaud S., Vadacchino M., Wang Kaige, Quantum Semiclass. Opt. 2, 159-187 (1990).

[12] Shen Y.R., The principles of nonlinear optics (Wiley, New-York, 1984)

[13] Yuen H.P. et Shapiro J.H. 1979, Optics Lett. 4, 334

[14] Polzik E. S., Carri J. et Kimble H.J., Appl. Phys. B 55, 279 (1992).

[15] Schneider K., Bruckmeier R., Hansen H., Schiller S. et Mlynek J., Opt. Lett. 21, 1396 (1996); Schneider K., Lang M., Mlynek J. et Schiller S., Opt. Express 2, 59 (1998).

[16] Shelby R.M., Levenson M.D, Perlmutter S.H., DeVoe R.G. et Walls D.F. 1986, Phys. Rev. Lett. 57, 691; Schumaker B.L., Perlmutter S.H., Shelby R.M. et Levenson M.D. 1987, Phys. Rev. Lett. 58, 357

[17] Fox A.M., Baumberg J.J., Dabbicco M., Huttner B. et Ryan J.F., Phys. Rev. Lett. 74, 1728 (1995)

[18] Rosenbluh M., Shelby R.M., Phys. Rev. Lett. 66153 (1991); Bergman K., Haus H.A., Opt. Lett, 16. 663 (1991); Bergman K. Doerr C.R., Haus H.A., Shirasaki M., , Opt. Lett. 18, 643-645 (1993).

[19] Raizen,M.G., Orozco L.A., Min Xiao, Boyd T.L. et Kimble H.J., Phys. Rev. Lett. 59, 198 (1987)

[20] Lambrecht A., Coudreau T,. Steinberg A.M. et Giacobino E., Europhys. Lett. 36, 93 (1996).

[21] Reid M.D. Phys. Rev. A 37, 4792 (1988); Castelli F., Lugiato L.A., Vadacchino M., Nuovo Cimento B 10, 183 (1988); Hilico L., Fabre C., Reynaud S., Giacobino E., Phys. Rev. A 46, 4397 (1992).

[22] Rarity J.G., Tapster P.R. et Jakeman E. Optics Commun. 62, 201 (1987); Rarity J.G. et Tapster P.R., in IQEC Technical Digest Series Vol. 8 (OSA, Washington D.C, 1990) p. 8; Abram I., Raj R.K., Oudar J.L. et Dolique G., Phys. Rev. Lett. 57, 2516 (1987); Aytur O. et Kumar P., Phys. Rev.Lett. 65, 1551 (1990)

[23] Reynaud S., Fabre C. et Giacobino E., J. Optical Society Am. B4, 1520 (1987); Fabre C., Giacobino E., Heidmann A. et Reynaud S., J. de Physique 50, 1209 (1989)

[24] Debuisschert T., Reynaud S., Heidmann A., Giacobino E. et Fabre C., Quantum Opt. 1, 3 (1989); Mertz J., Debuisschert T., Heidmann A., Fabre C., Giacobino E., Opt. Lett. 16 1234 (1991)

[25] Machida S. et Yamamoto Y., Phys Rev Lett. 60, 792 (1988); Richardson W.H., Yamamoto Y, 1991 Phys. Rev. Lett. 66, (1963); Richardson W.H., Machida S., Yamamoto Y, Phys. Rev. Lett. 66, 2867, (1991). 
[26] Freeman M. J., Wang H., Steel D.G., Craig R. et Scifres D.R., Opt. Lett. 18, 379 (1993); Kilper D.C., Steel D.G., Craig R. et Scifres D.R., Opt. Lett. 21, 1283 (1996).

[27] Friberg S.R., Machida S., Werner M.J., Levanon A. et Mukai T., Phys. Rev. Lett. 77, 3775-3778 (1996); Spälter S. Burk M, Strössner U., Böhm M., Sizmann A. et Leuchs G., Europhys. Lett. 38, 335-340 (1997).

[28] Nabors C.D. et Shelby R.M., Phys. Rev. A 42, 556 (1990); Marin F., Bramati A., Jost V. et Giacobino E., Optics Commun. 140, 146-157 (1997).

[29] Caves C.M., Phys. Rev. D 21, 1963 (1981); Min Xiao, Ling-An Wu et Kimble H.J., Phys. Rev. Lett. 59, 278 (1987).

[30] Bencheikh K., Simonneau C. et Levenson J.A., Phys. Rev. Lett. 78, 34 (1997). 